\title{
Development of Simultaneous Analysis of Thirteen Bioactive Compounds in So-Cheong-Ryong-Tang Using UPLC-DAD
}

\author{
Ji Hyun Jeong $\left(\mathbb{D}\right.$, Seon Yu Lee $\mathbb{D}^{D}$, Bo Na Kim $\mathbb{D}^{D}$, Guk Yeo Lee $\mathbb{D}^{D}$, and Seong Ho Ham $(\mathbb{D}$ \\ National Development Institute of Korean Medicine, Udae land gil 288, Jangheung-gun, Jeollanam-do 59338, Republic of Korea \\ Correspondence should be addressed to Seong Ho Ham; phd_ham@nikom.or.kr
}

Received 11 December 2017; Accepted 11 March 2018; Published 2 May 2018

Academic Editor: Pablo Richter

Copyright (c 2018 Ji Hyun Jeong et al. This is an open access article distributed under the Creative Commons Attribution License, which permits unrestricted use, distribution, and reproduction in any medium, provided the original work is properly cited.

So-Cheong-Ryong-Tang, which is a standardized Korean medicine of the National Health Insurance, is a traditional prescription for the treatment of allergic rhinitis, bronchitis, and bronchial asthma. Simultaneous analysis and development of SCRT is essential for its stability, efficacy, and risk management. In this study, a simple, reliable, and accurate method using ultrahighperformance liquid chromatography (UPLC) fingerprinting with a diode array detector (DAD) was developed for the simultaneous analysis. The chromatographic separation of the analytes was performed by an ACQUITY UPLC BEH C18 column $(1.7 \mu \mathrm{M}, 2.1 \times 100 \mathrm{~mm}$, Waters) with a mobile phase of water containing $0.01 \%$ (v/v) phosphoric acid and acetonitrile containing $0.01 \%(\mathrm{v} / \mathrm{v})$ phosphoric acid. The flow rate and detection wavelength were set at $0.4 \mathrm{~mL} / \mathrm{min}$ and $215,230,254$, and $280 \mathrm{~nm}$. All calibration curves of the thirteen components showed good linearity $\left(R^{2}>0.999\right)$. The limit of detection and limit of quantification ranged $0.001-0.360$ and $0.004-1.200 \mu \mathrm{g} / \mathrm{mL}$, respectively. The relative standard deviation (RSD) of intra- and interday was less than $2.60 \%$, and the recoveries were within the range $76.08-103.79 \%$ with an RSD value of $0.03-1.50 \%$. The results showed that the developed method was simple, reliable, accurate, sensitive, and precise for the quantification of bioactive components of SCRT.

\section{Introduction}

Traditional Korean medicines, because of their high effectiveness and low toxicity, have been used for thousands of years for the prevention and treatment of various kinds of human diseases. Various ingredients in these herbs cause the efficacy of traditional Korean medicines, which consist of many herbal combinations. Therefore, the consistency of the composition and proportion of the composition are the key to quality control in safety, efficacy, and risk management. In general, analyzing a single marker compound is simple and convenient, but it does not provide sufficient quantitative information on other components in Korean medicines. Thus, over the past decade, the chromatographic fingerprinting method has been considered one of the most important and acceptable approaches for the identification and quality evaluation of Korean medicines $[1,2]$. So-CheongRyong-Tang, standardized as a Korean medicine of the National Health Insurance, is a traditional prescription for the treatment of allergic rhinitis, bronchitis, and bronchial asthma [3]. Recently, SCRT was reported to show therapeutic effects in in vivo experiments on the respiratory system for allergic rhinitis and asthma [3-9]. So-CheongRyong-Tang (SCRT, Xiao-Qing-Long-Tang in Chinese, Shoseiryu-to in Japanese) is composed of eight herbal preparations (Ephedrae herba, Paeoniae radix, Glycyrrhizae radix, Zingiberis rhizoma, Cinnamomi ramulus, Schisandrae fructus, Pinelliae rhizoma, and Asiasari radix) $[4-6,8]$.

To optimize the quality control of SCRT, thirteen bioactive compounds from eight herbal preparations were chosen. Among the 13 standard compounds, ephedrine and catechin were found in Ephedrae herba, which is known for its efficacy as a sympathomimetic and its antiobesity effects [10]. Albiflorin, paeoniflorin, benzoic acid, PGG, and methyl gallate are the major constituents of Paeoniae radix, which has anti-inflammatory, analgesic, antispasmodic, liver protection, and immune regulatory functions $[11,12]$. Liquiritin, Liquiritin apioside, and Glycyrrhizin from Glycyrrhizae Radix were used, which is an effective detoxifying agent, presenting neuroprotective effect, antiviral activity, and 
<smiles>CN[C@@H](C)[C@H](O)c1ccccc1</smiles>

1. Ephedrine<smiles>COC(=O)c1cc(O)c(O)c(O)c1</smiles>

2. Methyl gallate<smiles>Oc1cc(O)c2c(c1)OC(c1ccc(O)c(O)c1)C(O)C2</smiles>

3. Catechin

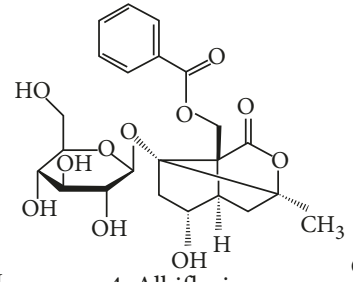

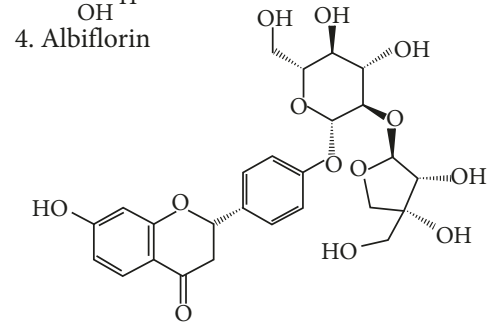

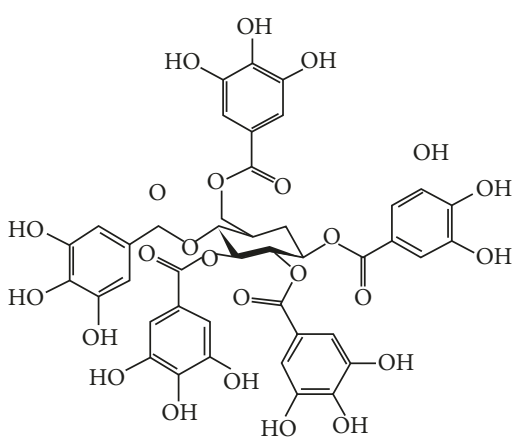

9. 1,2,3,4,6-Pentagalloyl glucose<smiles>O=C(O)c1ccccc1</smiles>

6. Benzoic acid

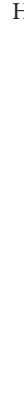

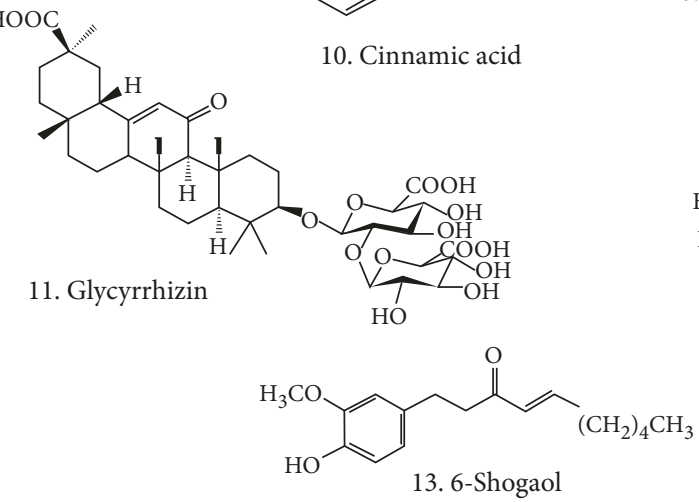

8. Liquiritin apioside<smiles>O=C(O)/C=C/c1ccccc1</smiles>

10. Cinnamic acid<smiles>COc1cc2c(c(OC)c1OC)-c1c(cc(OC)c(OC)c1OC)C[C@@](C)(O)[C@H](C)C2</smiles>

12. Schisandrin

Figure 1: Chemical structures of the 13 marker compounds.

anti-inflammatory, antitumor, and antibiosis effects [13]. 6-Shogaol from Zingiberis Rhizoma was found to have various pharmacological activities, including antioxidative, antitumorigenic, and immunomodulatory effects, and is an effective antimicrobial and antiviral agent [14]. Cinnamomi ramulus, which includes cinnamic acid, has been found to be able to effectively attenuate influenza virus, inflammations, human platelet aggregation, and arachidonic acid metabolism [15] and is known for its antimicrobial activity against [16]. The major constituent of Schisandrae fructus, which includes schisandrin, was found to have liver protective, hypoglycemic, antioxidant, antiaging, immune regulatory, antitumor, and bactericidal effects and plays a role in regulating the central nervous system [17]. Pinelliae rhizoma has antitussive, antiemetic, glandular secretion-inhibiting, and antitumor effects [18]. Asiasari radix has been used as an analgesic, antitussive, or antiallergic agent [19].

Several studies of these compounds have been developed for qualitative and quantitative analyses using highperformance liquid chromatography-diode array detector (HPLC-DAD) and mass spectrometry (HPLC-ESI-MS) $[4,5,9]$. However, these methods cannot offer simultaneous analysis of the multiple bioactive compounds in SCRT. Although an HPLC method for simultaneous determination of the four marker constituents of SCRT has been developed, there are limitations to the quantitative and qualitative analyses of many compounds in SCRT. Therefore, methods for simultaneously detecting these biomarkers in SCRT are essential to ensure efficient quality control and pharmaceutical evaluation. In this study, it was necessary to find more accurate, efficient, and stable solvent extraction conditions prior to simultaneous analysis. We performed simultaneous determination of the thirteen marker compounds.

\section{Experimental Materials and Reagents}

2.1. Chemicals and Reagents. Ephedrine, methyl gallate, catechin, albiflorin, paeoniflorin, benzoic acid, liquiritin, liquiritin apioside, 1,2,3,4,6-pentagalloyl glucose (PGG), cinnamic acid, glycyrrhizin, schisandrin, and 6-shogaol were purchased from Sigma-Aldrich Co. (St. Louis, MO, USA). The purity of all standards was $>97 \%$. Figure 1 shows the chemical structures of the thirteen bioactive compounds. HPLC-grade acetonitrile and methanol were purchased from J. T. Baker Inc. (Phillipsburg, NJ, USA). Deionized water was prepared using an ultrapure water production apparatus (Human Corporation, Seoul, Korea). SCRT medicines were purchased from Kyoungbang Medicinal Herbs (Incheon, Korea).

2.2. Preparation of Standard and Sample Solutions. Standard stock solutions of the thirteen bioactive standards-ephedrine, 


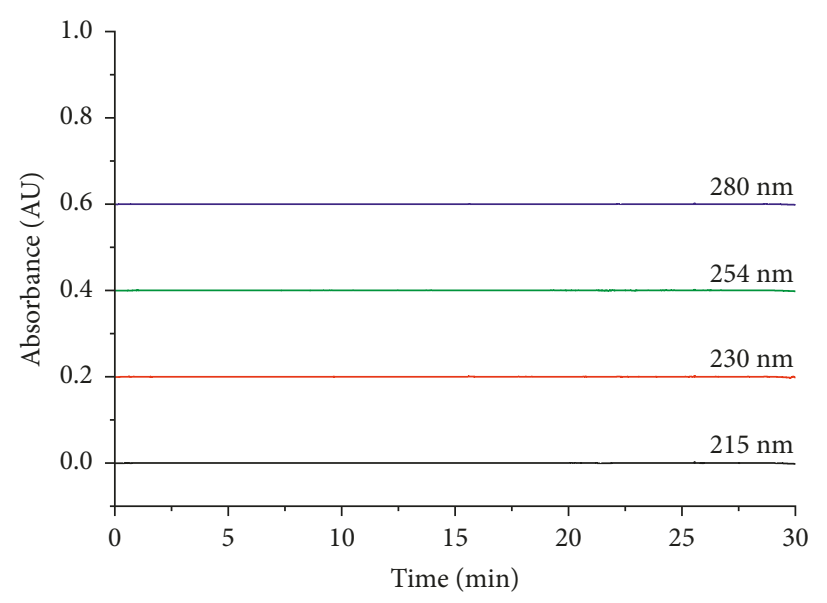

(a)

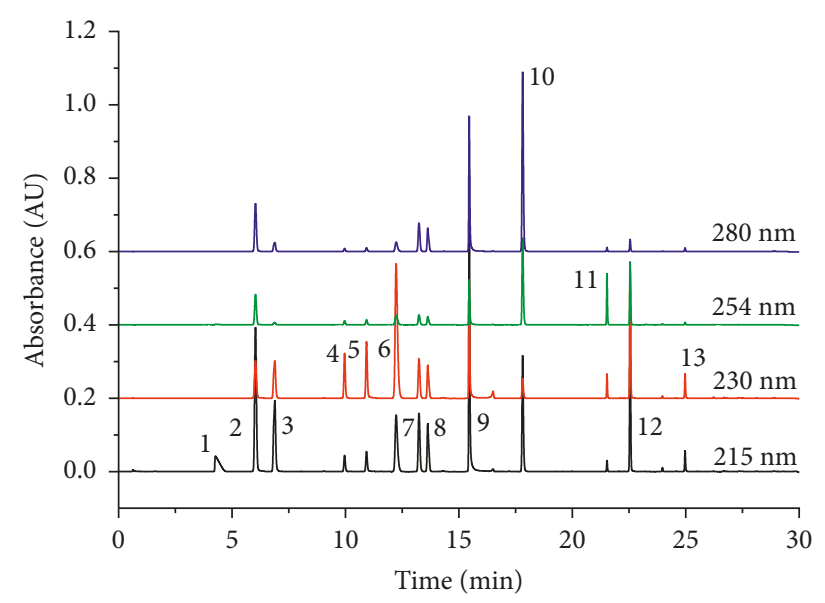

(b)

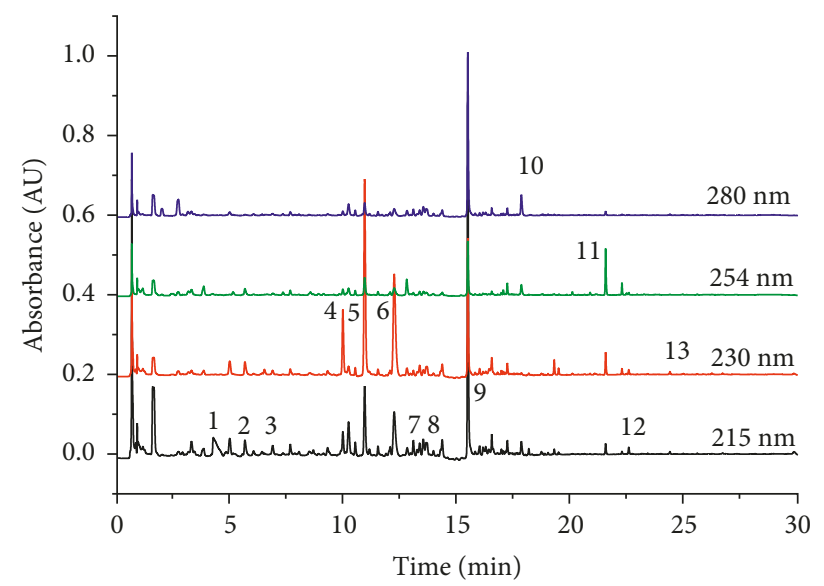

(c)

FIgURE 2: UPLC chromatograms of the (a) blank solvent, (b) standard mixture, and (c) SCRT extract.

methyl gallate, catechin, albiflorin, paeoniflorin, benzoic acid, liquiritin, liquiritin apioside, 1,2,3,4,6-pentagalloyl glucose (PGG), cinnamic acid, glycyrrhizin, schisandrin, and 6-shogaol-were prepared by accurately weighing appropriate amounts of reference compounds and dissolving in methanol. The thirteen bioactive standards were mixed in stock solutions and then diluted serially to seven concentrations for the construction of calibration curves. All the solutions were stored at $4^{\circ} \mathrm{C}$.

2.3. Extraction Method. The herbal medicine prepared by water extraction contains a water-soluble component and some lipoid-soluble substances, and most of the high molecular weight polymers are contained in a suspended state. It is necessary to estimate and optimize the extraction conditions for the 13 marker components in the SCRT sample. In this study, the liquid extraction method was selected, and aqueous methanol (20,50, and 80\%) was tried and examined as the extraction solvent to evaluate the optimal extraction solvent. Second, the volume (50 and $100 \mathrm{~mL}$ ) of the extraction solvent was investigated. Finally, extraction methods using ultrasonic or reflux were investigated [20-22].
2.4. Preparation of Sample Solution. The SCRT powder $(2.4 \mathrm{~g}$, 1 dose) was weighed precisely and extracted with $80 \%$ methanol-water (v/v) solution in an ultrasonic water bath for $10 \mathrm{~min}$ at room temperature. Then, the samples were refluxed twice at $80^{\circ} \mathrm{C}$ for $30 \mathrm{~min}$, followed by filtration and making up to volume in a volumetric flask. The samples were centrifuged $\left(4,000 \mathrm{rpm}, 10 \mathrm{~min}, 18^{\circ} \mathrm{C}\right)$, and the supernatant was filtered with a $0.2 \mu \mathrm{m}$ membrane filter, prior to injection. All working solutions and sample solutions were stored at $4^{\circ} \mathrm{C}$ before use.

2.5. Chromatographic Conditions by UPLC-DAD. The simultaneous determination of thirteen bioactive compounds in SCRT was performed on the UPLC-DAD system equipped with a pump, an autosampler, and a photodiode array detector, and the amount of data were calculated using Empower software. Chromatographic separation was carried out using an ACQUITY UPLC BEH C18 column $(1.7 \mu \mathrm{m}, 2.1 \times 100 \mathrm{~mm}$, Waters $)$, and the column temperature was kept at $40^{\circ} \mathrm{C}$. The mobile phase consisted of $(\mathrm{A})$ water (0.01\% phosphoric acid, v/v) and (B) acetonitrile $(0.01 \%$ phosphoric acid, v/v). The gradient solvent was optimized and performed as $98 \% \mathrm{~A}$ (0-1 min), $98-84 \% \mathrm{~A}$ (1-14 min), 

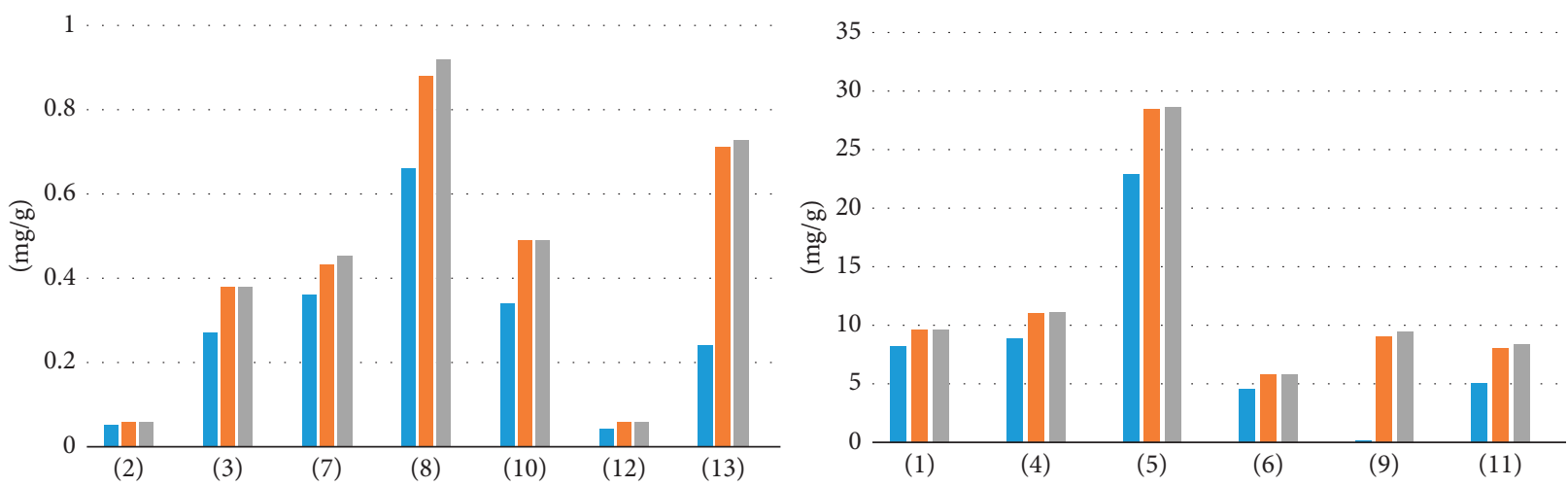

- $20 \% \mathrm{MeOH}$

$50 \% \mathrm{MeOH}$

- $80 \% \mathrm{MeOH}$

(a)
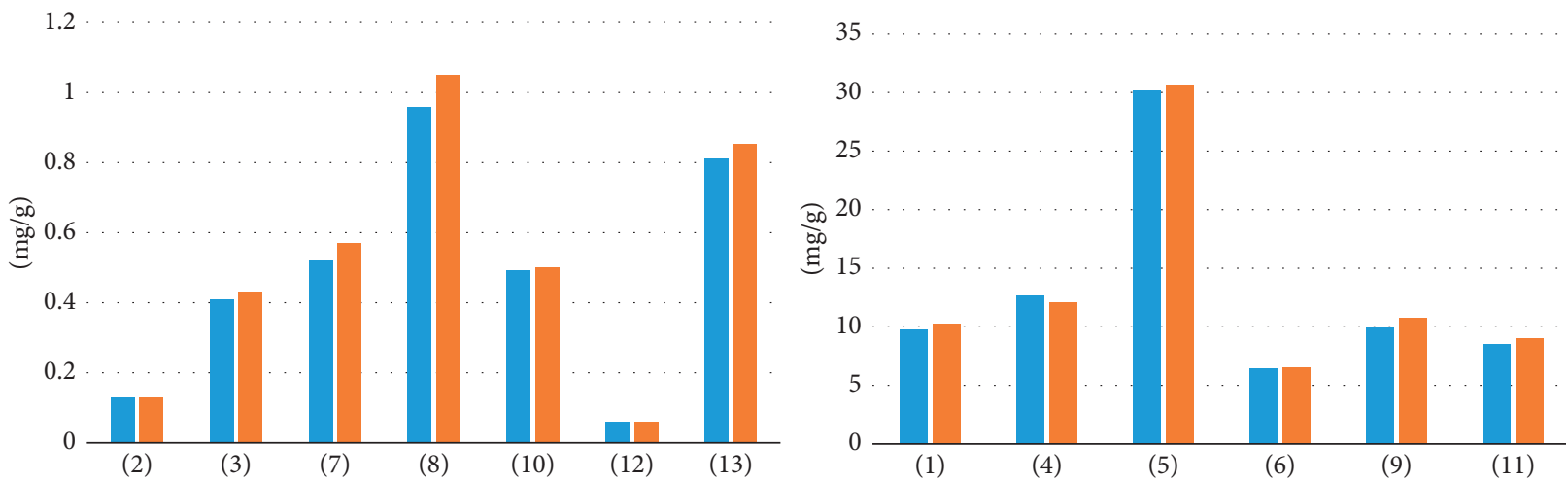

- $50 \mathrm{~mL}$

- $100 \mathrm{~mL}$

(b)
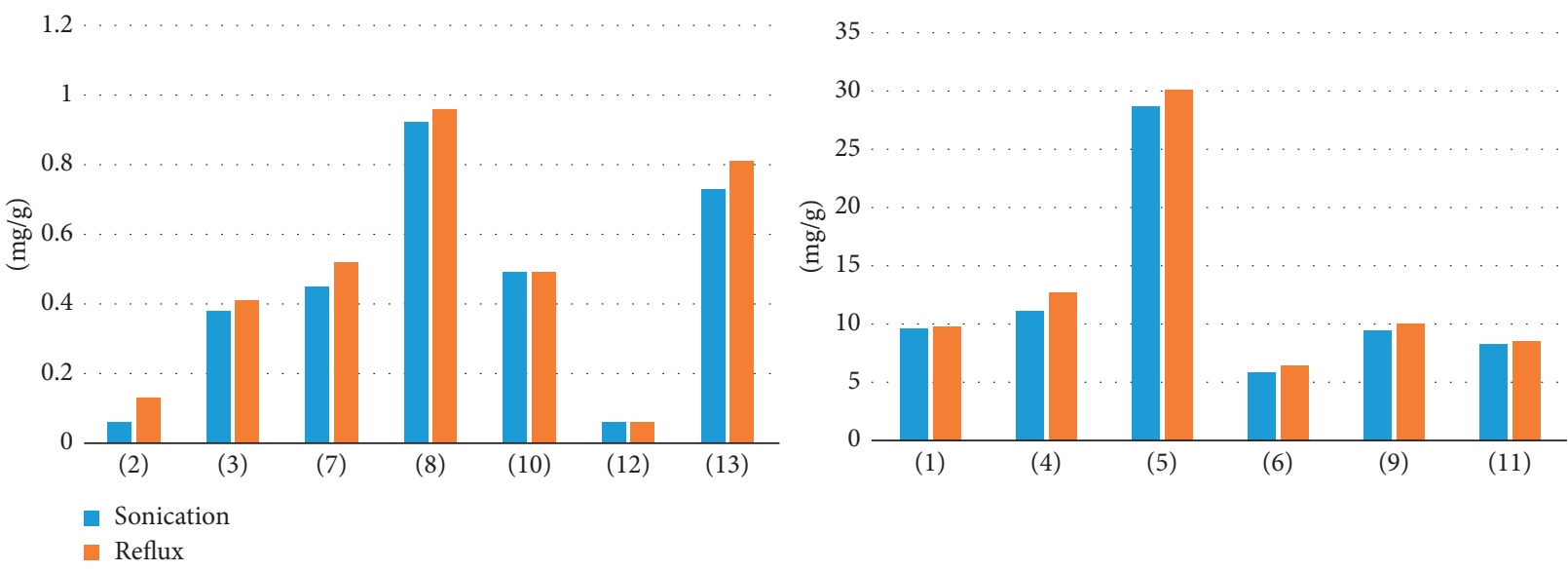

(c)

FIGURE 3: Efficiencies of the extraction for the nine compounds in SCRT using different (a) extraction solvents, (b) volumes of the extraction solvent, and (c) extraction methods.

84-60\% A (14-21 min), 60-20\% A (21-28 min), 20\% A (28$29 \mathrm{~min})$, and $20-98 \% \mathrm{~A}(29-29.5 \mathrm{~min})$, at a flow rate of $0.4 \mathrm{~mL} / \mathrm{min}$. The detection wavelengths for analytes were set at $215,230,254$, and $280 \mathrm{~nm}$, and the injection volume of each sample was $2 \mu \mathrm{L}$.
2.6. Method Validation. The method was validated for linearity, limit of detection (LOD), limit of quantification (LOQ), specificity, precision (interday, intraday, and repeatability), and accuracy (recovery), following the guideline on Bioanalytical Method Validation [23-26]. 

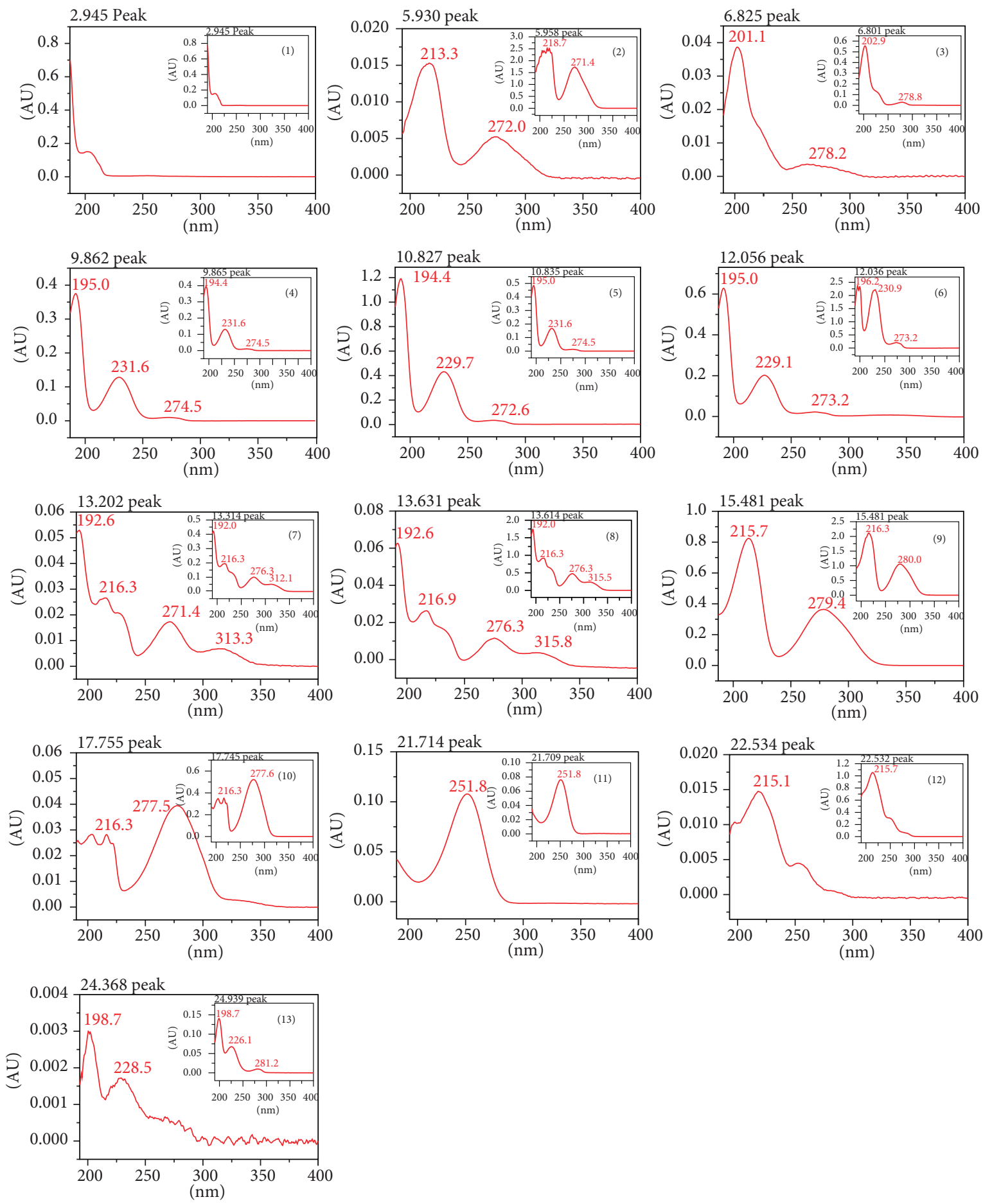

FIGURE 4: UV spectra of the thirteen marker compounds in SCRT corresponding to the standard solution (inset).

2.6.1. Linearity, Limits of Detection (LODs), and Limits of Quantification (LOQs). The standard solutions of the 13 compounds were prepared by serially diluting the stock solution to appropriate concentrations for plotting the calibration curves $[28,29]$. These solutions of the 13 compounds were analyzed in triplicate [28]. The calibration standard curve was plotted with the peak area ( $y$-axis) versus concentration ( $x$-axis) for each analyte in that range [30]. All calibration curves were required to have a correlation value of at least 0.995 . The limit of detection (LOD) and limit of quantification (LOQ) were determined on the basis of signal-to-noise ratio $(\mathrm{S} / \mathrm{N})$ :

$$
\begin{aligned}
& \mathrm{LOD}=\frac{\operatorname{amount} * 3.3}{(\mathrm{~S} / \mathrm{N})}, \\
& \mathrm{LOQ}=\frac{\operatorname{amount} * 10}{(\mathrm{~S} / \mathrm{N})} .
\end{aligned}
$$


TABLE 1: Regression data, LODs, and LOQs for the marker compounds analyzed by UPLC.

\begin{tabular}{|c|c|c|c|c|c|}
\hline & Compound & Regression equation & $R^{2}$ & LOQ $(\mu \mathrm{g} / \mathrm{mL})$ & $\mathrm{LOD}(\mu \mathrm{g} / \mathrm{mL})$ \\
\hline 1 & Ephedrine & $y=6,569.4 x-2,539.7$ & 0.9996 & 0.070 & 0.233 \\
\hline 2 & Methyl gallate & $y=49,631 x-14,901$ & 0.9995 & 0.007 & 0.026 \\
\hline 3 & Catechin & $y=28,138 x-4,098.4$ & 0.9992 & 0.008 & 0.029 \\
\hline 4 & Albiflorin & $y=6,199.8 x-2,341.7$ & 0.9996 & 0.040 & 0.134 \\
\hline 5 & Paeoniflorin & $y=8,506.1 x-1,169.5$ & 0.9994 & 0.018 & 0.062 \\
\hline 6 & Benzoic acid & $y=30,844 x-11,922$ & 0.9995 & 0.021 & 0.070 \\
\hline 7 & Liquiritin & $y=19,772 x-3,660$ & 0.9995 & 0.017 & 0.056 \\
\hline 8 & Liquiritin apioside & $y=15,249 x-1,630.9$ & 0.9996 & 0.145 & 0.483 \\
\hline 9 & $1,2,3,4,6$-Pentagalloyl glucose & $y=29,863 x-17,029$ & 0.9994 & 0.009 & 0.031 \\
\hline 10 & Cinnamic acid & $y=25,479 x-5,716.6$ & 0.9994 & 0.008 & 0.027 \\
\hline 11 & Glycyrrhizin & $y=3,546.4 x-422.55$ & 0.9994 & 0.073 & 0.244 \\
\hline 12 & Schisandrin & $y=86,584 x-4,328.7$ & 0.9995 & 0.001 & 0.004 \\
\hline 13 & 6-Shogaol & $y=1,025.4 x+1,176.6$ & 0.9988 & 0.360 & 1.200 \\
\hline
\end{tabular}

$R^{2}=$ correlation coefficient; $y=$ peak area; $x=$ sample concentration $(\mu \mathrm{g} / \mathrm{mL})$.

2.6.2. Precision, Accuracy, and Recovery. The precision of the method was evaluated by both intra- and interday tests. Three different concentrations of each biomarker in five replicates on the same day (intraday) and on three consecutive days (interday) were prepared to verify the precision and accuracy of the analytical method [30]. The precision was expressed as relative standard deviation (RSD, \%); a value of RSD within $\pm 15 \%$ is generally considered acceptable [28]. The accuracy of the assay is the closeness of the observed concentration to the nominal concentration [31]. The recoveries of analytes were determined by adding different concentrations of the 13 marker components into the SCRT sample solution $(2.4 \mathrm{mg} / \mathrm{mL})$. Recovery (\%) was calculated with the following equation:

$\operatorname{Recovery}(\%)=\frac{(\text { amount found }- \text { original amount })}{\text { amount spiked }} \times 100 \%$.

\section{Results and Discussion}

3.1. Optimization of Chromatographic Condition. A chromatogram of SCRT was obtained using an UPLC-PDA [4]. Figure 2 shows typical chromatograms corresponding to the mixed standard and SCRT. To obtain accurate, valid, and optimal separation, the UPLC conditions were investigated with regard to column, mobile phase (water-acetonitrile with different modifiers including acetic acid, formic acid, and phosphoric acid), detection wavelength $(215,230,254$, and $280 \mathrm{~nm})$, and mobile-phase flow rate $(0.5,0.4,0.3$, and $0.2 \mathrm{~mL} / \mathrm{min})$ [32] The best UPLC conditions were obtained from the ACQUITY UPLC BEH C18 column, which had better resolution than the others. The gradient solvent system consisted of $0.01 \%$ phosphoric acid in water (A) and $0.01 \%$ phosphoric acid in acetonitrile (B), at a column temperature of $40^{\circ} \mathrm{C}$, with a flow rate of $0.4 \mathrm{~mL} / \mathrm{min}$. Four detection wavelengths $(215,230,254$, and $280 \mathrm{~nm}$ ) were finally selected to achieve the goal of high detection sensitivity and small interference because the maximum absorption of the 13 reference compounds was different.

3.2. Optimization of the Sample Extraction Protocol. The extraction conditions, for example, extraction solvent, method, and volume, can easily influence the efficiency of the extraction. In this paper, aqueous methanol (20,50, and $80 \%$ ) was examined as the extraction solvent for SCRT by ultrasonication for $30 \mathrm{~min}$ [33]. Figure 3(a) shows the results, which indicate that $80 \%$ methanol was the best extraction solvent. Second, the volume of solvent (50 and $100 \mathrm{~mL}$ ) was investigated, and Figure 3(b) shows the results. The volume of the extraction solvent was $100 \mathrm{~mL}$. The extraction efficiency was better at $100 \mathrm{~mL}$ volume in lowcontent compounds. Finally, Figure 3(c) shows the results of the extraction method using ultrasound and reflux, which indicate that the extraction method using reflux showed high efficiency. In the end, the suitable extraction conditions were as follows: the samples were extracted by reflux with $80 \%$ methanol in a volume of $100 \mathrm{~mL}$.

\subsection{Method Validation}

3.3.1. Specificity. The specificity was determined by comparing the peak purity of the 13 markers with the extracted samples and the standard. Figure 4 shows the UV spectra of individual marker compounds, which confirm that the peaks are pure and there is no interference from the impurities [34].

3.3.2. Linearity, $L O D$, and $L O Q$. Table 1 summarizes the calibration curves, LOQ, and LOD of the thirteen analytes. The linearity of the developed method was assessed using seven concentrations: ephedrine, albiflorin, paeoniflorin, benzoic acid, PGG, glycyrrhizin $(0.1 \sim 100 \mu \mathrm{g} / \mathrm{mL})$, methyl gallate, catechin, liquiritin, liquiritin apioside, cinnamic acid $(0.05 \sim 50 \mu \mathrm{g} / \mathrm{mL})$, and schisandrin $(0.02 \sim 20 \mu \mathrm{g} / \mathrm{mL})$ with correlation coefficients $R^{2} \geq 0.999$. The values of LOD and LOQ were in the ranges of $0.001-0.360 \mu \mathrm{g} / \mathrm{mL}$ and $0.004-1.200 \mu \mathrm{g} / \mathrm{mL}$, respectively. The results showed that the calibration curves were within the adequate range and exhibited good sensitivity for the analysis of the thirteen bioactive components [28].

3.3.3. Precision, Accuracy, and Recovery. Table 2 shows the results of the intra- and interday precision tests. The values of RSD (\%) for intra- and interday tests were within the 
TABLE 2: Precision (intra- and interday) and accuracy of the thirteen analytes.

\begin{tabular}{|c|c|c|c|c|c|c|}
\hline \multirow{2}{*}{$\begin{array}{l}\text { Analyte } \\
\text { concentration } \\
(\mu \mathrm{g} / \mathrm{mL})\end{array}$} & \multicolumn{3}{|c|}{ Intraday $(n=5)$} & \multicolumn{3}{|c|}{ Interday $(n=3)$} \\
\hline & $\begin{array}{c}\text { Measured amount } \\
(\text { mean } \pm \mathrm{SD}, \mathrm{Dg} / \mathrm{mL})\end{array}$ & RSD (\%) & Accuracy (\%) & $\begin{array}{c}\text { Measured amount } \\
(\text { mean } \pm \mathrm{SD}, \mathrm{Dg} / \mathrm{mL})\end{array}$ & RSD (\%) & Accuracy (\%) \\
\hline \multicolumn{7}{|l|}{ Ephedrine } \\
\hline 25 & $5.03 \pm 0.02$ & 0.43 & 100.59 & $4.97 \pm 0.04$ & 0.82 & 99.30 \\
\hline 50 & $25.59 \pm 0.01$ & 0.03 & 102.36 & $25.33 \pm 0.23$ & 0.90 & 101.31 \\
\hline 100 & $100.46 \pm 0.08$ & 0.08 & 100.46 & $100.46 \pm 0.31$ & 0.31 & 100.46 \\
\hline \multicolumn{7}{|l|}{ Methyl gallate } \\
\hline 2.5 & $2.48 \pm 0.04$ & 1.49 & 99.36 & $2.51 \pm 0.01$ & 0.35 & 100.57 \\
\hline 12.5 & $12.29 \pm 0.00$ & 0.03 & 98.34 & $12.44 \pm 0.12$ & 1.00 & 99.48 \\
\hline 50 & $49.14 \pm 0.31$ & 0.63 & 98.27 & $50.03 \pm 1.00$ & $\begin{array}{l}1.00 \\
1.99\end{array}$ & 100.06 \\
\hline \multicolumn{7}{|l|}{ Catechin } \\
\hline 2.5 & $2.53 \pm 0.00$ & 0.12 & 101.09 & $2.52 \pm 0.02$ & 0.75 & 100.65 \\
\hline 12.5 & $12.50 \pm 0.00$ & 0.02 & 100.00 & $12.50 \pm 0.00$ & 0.02 & 100.02 \\
\hline 50 & $49.69 \pm 0.21$ & 0.43 & 99.38 & $50.14 \pm 0.48$ & 0.96 & 100.28 \\
\hline \multicolumn{7}{|l|}{ Albiflorin } \\
\hline 5 & $5.02 \pm 0.03$ & 0.61 & 100.36 & $5.12 \pm 0.07$ & 1.42 & 102.42 \\
\hline 25 & $25.05 \pm 0.04$ & 0.15 & 100.20 & $25.12 \pm 0.11$ & 0.43 & 100.47 \\
\hline 100 & $98.68 \pm 0.16$ & 0.16 & 98.68 & $99.78 \pm 0.80$ & 0.80 & 99.78 \\
\hline \multicolumn{7}{|l|}{ Paeoniflorin } \\
\hline 5 & $5.05 \pm 0.10$ & 1.94 & 101.10 & $5.13 \pm 0.04$ & 0.73 & 102.62 \\
\hline 25 & $25.24 \pm 0.08$ & 0.30 & 100.96 & $25.11 \pm 0.27$ & 1.07 & 100.44 \\
\hline 100 & $101.46 \pm 1.96$ & 0.93 & 101.46 & $100.46 \pm 0.15$ & 0.15 & 100.46 \\
\hline \multicolumn{7}{|l|}{ Benzoic acid } \\
\hline 1 & $1.01 \pm 0.01$ & 1.35 & 100.92 & $1.01 \pm 0.02$ & 1.61 & 101.28 \\
\hline 5 & $5.02 \pm 0.02$ & 0.39 & 100.31 & $4.99 \pm 0.04$ & 0.80 & 99.80 \\
\hline 100 & $96.82 \pm 2.43$ & 2.51 & 96.82 & $100.59 \pm 0.28$ & 0.28 & 100.59 \\
\hline \multicolumn{7}{|l|}{ Liquiritin } \\
\hline 2.5 & $2.49 \pm 0.01$ & 0.57 & 99.64 & $2.44 \pm 0.04$ & 1.64 & 97.67 \\
\hline 12.5 & $12.14 \pm 0.01$ & 0.05 & 97.10 & $12.37 \pm 0.21$ & 1.66 & 98.99 \\
\hline 50 & $50.20 \pm 0.29$ & 0.58 & 100.39 & $50.17 \pm 0.48$ & 0.96 & 100.35 \\
\hline \multicolumn{7}{|c|}{ Liquiritin apioside } \\
\hline 2.5 & $2.53 \pm 0.03$ & 1.08 & 101.11 & $2.51 \pm 0.00$ & 0.16 & 100.45 \\
\hline 12.5 & $12.56 \pm 0.00$ & 0.02 & 100.48 & $12.51 \pm 0.04$ & 0.34 & 100.07 \\
\hline 50 & $49.91 \pm 0.36$ & 0.72 & 99.81 & $49.95 \pm 0.09$ & 0.17 & 99.90 \\
\hline \multicolumn{7}{|c|}{ 1,2,3,4,6-Pentagalloyl glucose } \\
\hline 5 & $4.94 \pm 0.07$ & 1.45 & 98.73 & $5.05 \pm 0.03$ & 0.68 & 101.00 \\
\hline 25 & $25.26 \pm 0.02$ & 0.08 & 101.04 & $25.15 \pm 0.10$ & 0.38 & 100.60 \\
\hline 100 & $99.89 \pm 0.45$ & 0.45 & 99.89 & $100.27 \pm 0.37$ & 0.37 & 100.27 \\
\hline \multicolumn{7}{|l|}{ Cinnamic acid } \\
\hline 0.5 & $0.50 \pm 0.00$ & 0.38 & 100.16 & $0.50 \pm 0.00$ & 0.33 & 99.45 \\
\hline 12.5 & $12.50 \pm 0.00$ & 0.02 & 99.96 & $12.51 \pm 0.02$ & 0.13 & 100.06 \\
\hline 50 & $50.03 \pm 0.01$ & 0.02 & 100.06 & $50.15 \pm 0.19$ & 0.38 & 100.31 \\
\hline \multicolumn{7}{|l|}{ Glycyrrhizin } \\
\hline 5 & $5.03 \pm 0.02$ & 0.30 & 100.62 & $4.99 \pm 0.11$ & 2.27 & 99.84 \\
\hline 25 & $25.04 \pm 0.11$ & 0.42 & 100.14 & $25.09 \pm 0.01$ & 0.05 & 100.35 \\
\hline 100 & $100.63 \pm 0.30$ & 0.29 & 100.63 & $100.65 \pm 0.17$ & 0.17 & 100.65 \\
\hline \multicolumn{7}{|l|}{ Schisandrin } \\
\hline 1 & $1.00 \pm 0.00$ & 0.11 & 100.03 & $1.00 \pm 0.00$ & 0.40 & 100.40 \\
\hline 5 & $5.09 \pm 0.00$ & 0.01 & 101.83 & $5.04 \pm 0.05$ & 0.90 & 100.89 \\
\hline 20 & $19.33 \pm 0.42$ & 2.17 & 96.64 & $20.12 \pm 0.18$ & 0.87 & 100.60 \\
\hline \multicolumn{7}{|l|}{ 6-Shogaol } \\
\hline 2 & $2.04 \pm 0.04$ & 2.00 & 101.93 & $2.05 \pm 0.05$ & 2.60 & 102.50 \\
\hline 50 & $50.36 \pm 0.04$ & 0.09 & 100.71 & $50.27 \pm 0.18$ & 0.35 & 100.54 \\
\hline 200 & $197.59 \pm 0.09$ & 0.05 & 98.79 & $199.67 \pm 1.72$ & 0.86 & 99.83 \\
\hline
\end{tabular}

$\mathrm{SD}=$ standard deviation; RSD $(\%)=(\mathrm{SD} /$ mean $) \times 100$; accuracy $(\%)=\left(C_{\mathrm{obs}} / C_{\text {nom }}\right) \times 100$. 
TABle 3: Determination of recoveries of the 13 compounds (1-13) in SCRT.

\begin{tabular}{|c|c|c|c|c|}
\hline Compounds & Spiked amount & Measured amount & Recovery (\%) & RSD (\%) \\
\hline \multirow{3}{*}{ Ephedrine } & 25 & $25.20 \pm 0.19$ & 100.78 & 0.73 \\
\hline & 50 & $51.86 \pm 0.78$ & 103.72 & 1.50 \\
\hline & 100 & $100.23 \pm 0.59$ & 100.23 & 0.59 \\
\hline \multirow{3}{*}{ Methyl gallate } & 5 & $4.47 \pm 0.01$ & 89.33 & 0.30 \\
\hline & 12.5 & $10.94 \pm 0.04$ & 87.53 & 0.33 \\
\hline & 50 & $45.63 \pm 0.03$ & 91.26 & 0.07 \\
\hline \multirow{3}{*}{ Catechin } & 5 & $4.98 \pm 0.02$ & 99.53 & 0.40 \\
\hline & 12.5 & $11.42 \pm 0.09$ & 91.39 & 0.76 \\
\hline & 50 & $45.53 \pm 0.30$ & 91.05 & 0.65 \\
\hline \multirow{3}{*}{ Albiflorin } & 5 & $5.15 \pm 0.02$ & 103.09 & 0.36 \\
\hline & 25 & $25.90 \pm 0.11$ & 103.59 & 0.42 \\
\hline & 100 & $98.23 \pm 1.06$ & 98.23 & 1.08 \\
\hline \multirow{3}{*}{ Paeoniflorin } & 10 & $10.05 \pm 0.02$ & 100.54 & 0.21 \\
\hline & 25 & $24.64 \pm 0.08$ & 98.55 & 0.34 \\
\hline & 100 & $100.97 \pm 0.35$ & 100.97 & 0.35 \\
\hline \multirow{3}{*}{ Benzoic acid } & 25 & $24.43 \pm 0.09$ & 97.70 & 0.39 \\
\hline & 50 & $47.29 \pm 0.17$ & 94.59 & 0.37 \\
\hline & 100 & $97.28 \pm 0.10$ & 97.28 & 0.10 \\
\hline \multirow{3}{*}{ Liquiritin } & 5 & $5.05 \pm 0.05$ & 100.93 & 0.91 \\
\hline & 12.5 & $12.54 \pm 0.12$ & 100.31 & 0.97 \\
\hline & 50 & $42.98 \pm 0.22$ & 85.96 & 0.52 \\
\hline \multirow{3}{*}{ Liquiritin apioside } & 5 & $4.94 \pm 0.04$ & 98.79 & 0.79 \\
\hline & 12.5 & $12.55 \pm 0.03$ & 100.43 & 0.27 \\
\hline & 50 & $48.99 \pm 0.45$ & 97.98 & 0.93 \\
\hline \multirow{3}{*}{ 1,2,3,4,6-Pentagalloyl glucose } & 10 & $10.00 \pm 0.13$ & 99.98 & 1.30 \\
\hline & 50 & $46.89 \pm 0.25$ & 93.77 & 0.53 \\
\hline & 100 & $99.17 \pm 0.56$ & 99.17 & 0.56 \\
\hline \multirow{3}{*}{ Cinnamic acid } & 12.5 & $12.61 \pm 0.06$ & 100.88 & 0.49 \\
\hline & 25 & $24.70 \pm 0.02$ & 98.81 & 0.09 \\
\hline & 50 & $52.28 \pm 0.22$ & 104.57 & 0.42 \\
\hline \multirow{3}{*}{ Glycyrrhizin } & 25 & $24.13 \pm 0.16$ & 96.54 & 0.66 \\
\hline & 50 & $50.36 \pm 0.14$ & 100.72 & 0.27 \\
\hline & 100 & $102.79 \pm 0.52$ & 102.79 & 0.50 \\
\hline \multirow{3}{*}{ Schisandrin } & 1 & $0.98 \pm 0.01$ & 97.78 & 0.87 \\
\hline & 5 & $4.65 \pm 0.04$ & 92.93 & 0.77 \\
\hline & 20 & $17.98 \pm 0.03$ & 89.90 & 0.18 \\
\hline \multirow{3}{*}{ 6-Shogaol } & 20 & $15.22 \pm 0.02$ & 76.08 & 0.14 \\
\hline & 100 & $85.23 \pm 0.05$ & 85.23 & 0.06 \\
\hline & 200 & $177.66 \pm 0.05$ & 88.83 & 0.03 \\
\hline
\end{tabular}

ranges of $0.01-2.51 \%$ and $0.02-2.60 \%$, with accuracy from 96.82 to $102.36 \%$ and from 97.67 to $102.62 \%$, respectively [2]. The results indicated that the accuracy and precision of the proposed method were accurate and reliable for determination of the thirteen compounds in the sample of SCRT [28]. The average recovery (\%) of the 13 marker compounds shows the accuracy of this analytical method. The recovery ranged from 76.08 to $103.79 \%$, and the values of RSD were in the range $0.03-1.50 \%$. Table 3 summarizes these results; they indicate that the proposed method enables highly accurate simultaneous analysis of the thirteen compounds [35].

\section{Discussion}

Standardization and analysis of the marker compounds in herbal medicines are necessary for safety, efficacy, and risk management. A simple, reliable, and accurate method using ultrahigh-performance liquid chromatography (UPLC) fingerprinting with a diode array detector (DAD) was developed for the simultaneous qualitative and quantitative analyses of the thirteen biomarkers: ephedrine, methyl gallate, catechin, albiflorin, paeoniflorin, benzoic acid, liquiritin, liquiritin apioside, 1,2,3,4,6-pentagalloyl glucose (PGG), cinnamic acid, glycyrrhizin, schisandrin, and 6-shogaol in SCRT.

To optimize the quality control of SCRT, thirteen bioactive compounds from eight herbal preparations (Ephedrae herba, Paeoniae radix, Glycyrrhizae radix, Zingiberis rhizoma, Cinnamomi ramulus, Schisandrae fructus, Pinelliae rhizoma, and Asiasari radix) were chosen.

However, Pinelliae rhizoma and Asiasari radix were not detected in the SCRT extract sample. Because the contents of bioactive compounds in herbal medicines can differ as a function of the collection period, region, species, and preparation method, or the medicine contains a concentration 
lower than the LOD value, further studies should be required to perform quantification and qualification analyses, using a standard addition method or ultrahigh-performance liquid chromatography-tandem mass spectrometry (UPLC-MS/MS).

The results show that all biomarker components were detected (Figure 3), and identified based on the UV absorbance spectra (Figure 4) and retention times, by comparison with standard compounds. The UPLC-DAD method was validated, and the results showed good linearity, LOD, LOQ, precision, and accuracy, with RSD $<2.51 \%$. Furthermore, the method did not interfere with other chemical constituents in SCRT. The results show that our method is accurate and reliable for quantification and qualification of the bioactive components of SCRT.

\section{Conclusion}

The developed UPLC-DAD fingerprinting method for the simultaneous determination of thirteen biomarkers in SCRT, which include ephedrine, methyl gallate, catechin, albiflorin, paeoniflorin, benzoic acid, liquiritin, liquiritin apioside, PGG, cinnamic acid, glycyrrhizin, schisandrin, and 6-shogaol, proved an efficient tool for quality control and pharmaceutical evaluation. The results demonstrated that the developed UPLC-DAD method is simple, reliable, accurate, sensitive, and precise for quantification and qualification analyses of SCRT.

\section{Conflicts of Interest}

The authors declare that there are no conflicts of interest regarding the publication of this paper.

\section{Acknowledgments}

This paper was supported by a grant from the Modernization Project of Korean Medicinal Preparations and the National Development Institute of Korean Medicine (NICOM) and by a grant from the Ministry of Health and Welfare, Republic of Korea.

\section{References}

[1] D. K. Sharma, S. G. Kim, R. Lamichhane, K. H. Lee, A. Poudel, and H. J. Jung, "Development of UPLC fingerprint with multicomponent quantitative analysis for quality consistency evaluation of herbal medicine "hyangsapyeongwisan"," Journal of Chromatographic Science, vol. 54, no. 4, pp. 536-546, 2016.

[2] L. Zheng and D. Dong, "Development and validation of an HPLC method for simultaneous determination of nine active components in "Da-Chai-Hu-Tang"," Chinese Medicine, vol. 2, no. 1, pp. 20-28, 2011.

[3] C. Y. Lim, H. W. Kim, B. Y. Kim, and S. I. Cho, "Genome wide expression analysis of the effect of Socheongryong Tang in asthma model of mice," Journal of Traditional Chinese Medicine, vol. 35, no. 2, pp. 168-174, 2015.

[4] M. Y. Lee, C. S. Seo, J. Y. Kim, and H. K. Shin, "Evaluation of a water extract of So-Cheong-Ryong-Tang for acute toxicity and genotoxicity using in vitro and in vivo tests," $B M C$ Complementary and Alternative Medicine, vol. 15, no. 1, pp. 1-8, 2015.
[5] N. H. Yim, A. Kim, Y. P. Jung, T. Kim, C. J. Ma, and J. Y. Ma, "Fermented So-Cheong-Ryong-Tang (FCY) induces apoptosis via the activation of caspases and the regulation of MAPK signaling pathways in cancer cells," BMC Complementary and Alternative Medicine, vol. 15, no. 1, pp. 1-11, 2015.

[6] E. Ko, S. Rho, C. Cho et al., "So-Cheong-Ryong-Tang, tradititional Korean medicine, suppresses Th2 lineage development," Biological \& Pharmaceutical Bulletin, vol. 27, no. 5, pp. 739-743, 2004.

[7] H. W. Kim, C. Y. Lim, B. Y. Kim, and S. I. Cho, "So-CheongRyong-Tang, a herbal medicine, modulates inflammatory cell infiltration and prevents airway remodeling via regulation of interleukin-17 and GM-CSF in allergic asthma in mice," Pharmacognosy Magazine, vol. 10, no. 39, pp. 506-511, 2014.

[8] C. Park, S. H. Hong, G. Y. Kim, and Y. H. Choi, "So-CheongRyong-Tang induces apoptosis through activation of the intrinsic and extrinsic apoptosis pathways, and inhibition of the PI3K/Akt signaling pathway in non-small-cell lung cancer A549 cells," BMC Complementary and Alternative Medicine, vol. 15, no. 1, pp. 1-13, 2015.

[9] M. K. Lee, K. Y. Lee, J. Park, and S. H. Sung, "Simultaneous determination of paeoniflorin, trans-cinnamic acid, schisandrin and glycyrrhizin in So-Cheong-Ryong-Tang by HPLC-DAD and HPLC-ESI-MS," Natural Product Sciences, vol. 16 , no. 1 , pp. $26-31,2010$.

[10] H. J. Kim, J. M. Park, J. A. Kim, and B. P. Ko, "Effect of herbal Ephedra sinica and Evodia rutaecarpa on body composition and resting metabolic rate: a randomized, double-blind clinical trial in Korean premenopausal women," Journal of Acupuncture and Meridian Studies, vol. 1, no. 2, pp. 128-138, 2008.

[11] C. Kim, J. H. Lee, W. Kim, and S. K. Kim, "The suppressive effects of Cinnamomi Cortex and its phytocompound coumarin on oxaliplatin-induced neuropathic cold allodynia in rats," Molecules, vol. 21, no. 9, pp. 1-12, 2016.

[12] X. Li, W. Wang, Y. Su, Z. Yue, and J. Bao, "Inhibitory effect of an aqueous extract of Radix Paeoniae Alba on calcium oxalate nephrolithiasis in a rat model," Renal Failure, vol. 39, no. 1, pp. 120-129, 2017.

[13] D. Wu, J. Chen, H. Zhu, and X. Huang, "UPLC-PDA determination of paeoniflorin in rat plasma following the oral administration of Radix Paeoniae Alba and its effects on rats with collagen-induced arthritis," Experimental and Therapeutic Medicine, vol. 7, no. 1, pp. 209-217, 2013.

[14] X. Li, W. Chen, and D. Chen, "Protective effect against hydroxyl-induced DNA damage and antioxidant activity of radix glycyrrhizae (liquorice root)," Advanced Pharmaceutical Bulletin, vol. 3, no. 1, pp. 167-173, 2013.

[15] S. Chrubasik, M. H. Pittler, and B. D. Roufogalis, "Zingiberis rhizoma : a comprehensive review on the ginger effect and efficacy profiles," Phytomedicine, vol. 12, no. 9, pp. 684-701, 2005.

[16] M. T. Liang, C. H. Yang, S. T. Li et al., "Antibacterial and antioxidant properties of Ramulus Cinnamomi using supercritical CO2 extraction," European Food Research and Technology, vol. 227, no. 5, pp. 1387-1396, 2008.

[17] Z. Hou, G. Xu, X. Han, and L. An, "Pharmacological research of Schisandra chinensis (Turcz.) Baill," in Proceedings of ASME International Manufacturing Science and Engineering, pp. 116-119, Los Angeles, CA, USA, June 2015.

[18] X. Zhang, Y. Cai, L. Wang, H. Liu, and X. Wang, "Optimization of processing technology of Rhizoma Pinelliae Praeparatum and its anti-tumor effect," African Health Sciences, vol. 15, no. 1, pp. 101-106, 2015.

[19] J. B. Park, J. E. Lee, S. H. Jin, Y. Ko, and S. H. Jeong, "Evaluation of the effects of a low dose of Asiasari radix on 
stem cell morphology and proliferation," Journal of Korean Medicine, vol. 37, no. 2, pp. 85-92, 2016.

[20] M. Li, X. F. Hou, J. Zhang, S. C. Wang, Q. Fu, and L. C. He, "Applications of HPLC/MS in the analysis of traditional Chinese medicines," Journal of Pharmaceutical Analysis, vol. 1, no. 2, pp. 81-91, 2011.

[21] A. A. Boligon, R. B. Freitas, T. F. Brum, and L. F. Bauermann, "Antiulcerogenic activity of Scutia buxifolia on gastric ulcers induced by ethanol in rats," Acta Pharmaceutica Sinica B, vol. 4, no. 5, pp. 358-367, 2014.

[22] A. Bahrami, F. Ghamari, Y. Yamini, F. G. Shahna, and A. Moghimbeigi, "Hollow fiber supported liquid membrane extraction combined with HPLC-UV for simultaneous preconcentration and determination of urinary hippuric acid and mandelic acid," Membranes, vol. 7, no. 1, pp. 1-13, 2017.

[23] L. Y. Ma, Y. B. Zhang, Q. L. Zhou, Y. F. Yang, and X. W. Yang, "Simultaneous determination of eight ginsenosides in rat plasma by liquid chromatography-electrospray ionization tandem mass spectrometry; application to their pharmacokinetics," Molecules, vol. 20, no. 12, pp. 21597-21608, 2015.

[24] L. Yi, L. W. Qi, P. Li, Y. H. Ma, Y. J. Luo, and H. Y. Li, "Simultaneous determination of bioactive constituents in Danggui Buxue Tang for quality control by HPLC coupled with a diode array detector, an evaporative light scattering detector and mass spectrometry," Analytical and Bioanalytical Chemistry, vol. 389, no. 2, pp. 571-580, 2007.

[25] H. Bae, G. K. Jayaprakasha, J. Jifon, and B. S. Patil, "Extraction efficiency and validation of an HPLC method for flavonoid analysis in peppers," Food Chemistry, vol. 130, no. 3, pp. 751-758, 2012.

[26] D. Q. Tang, X. X. Zheng, X. Chen, D. Z. Yang, and Q. Du, "Quantitative and qualitative analysis of common peaks in chemical fingerprint of Yuanhu Zhitong tablet by HPLCDAD-MS/MS," Journal of Pharmaceutical Analysis, vol. 4, no. 2, pp. 96-106, 2014.

[27] J. H. Kim, C. S. Seo, S. S. Kim, and H. K. Shin, "Quality assessment of Ojeok-San, a traditional herbal formula, using high-performance liquid chromatography combined with chemometric analysis," Journal of Analytical Methods in Chemistry, vol. 2015, Article ID 607252, 11 pages, 2015.

[28] H. J. Yang, N. H. Yim, K. J. Lee, and J. Y. Ma, "Simultaneous determination of nine bioactive compounds in Yijin-tang via high-performance liquid chromatography and liquid spectrometry," Integrative Medicine Research, vol. 5, no. 2, pp. 140-150, 2016.

[29] Y. Wang, Q. Lin, and F. Ikegami, "Development and validation of an HPLC-DAD method for the simultaneous quantification of 8 characteristic components in kakkonoto decotion," Journal of Traditional Medicine, vol. 29, no. 4, pp. 195-202, 2012.

[30] L. H. Shaw, L. C. Lin, and T. H. Tsai, "HPLC-MS/MS analysis of a traditional Chinese medical formulation of Bu-YangHuan-Wu-Tang and its pharmacokinetics after oral administration to rats," Pharmacokinetics of Herbal Formulation, vol. 7, no. 8, pp. 1-13, 2012.

[31] Z. Yu, X. Gao, H. Yuan, and K. Bi, "Simultaneous determination of safflor yellow A, puerarin, daidzein, ginsenosides ( $\mathrm{Rg} 1, \mathrm{Rb} 1, \mathrm{Rd}$ ), and notoginsenoside $\mathrm{R} 1$ in rat plasma by liquid chromatography-mass spectrometry," Journal of Pharmaceutical and Biomedical Analysis, vol. 45, no. 2, pp. 327-336, 2007.

[32] A. Poudel, S. G. Kim, R. Lamichhane, Y. K. Kim, H. K. Jo, and H. J. Jung, "Quantitative assessment of traditional oriental herbal formulation samhwangsasim-tang using UPLC technique," Journal of Chromatographic Science, vol. 52, no. 2, pp. 176-185, 2014.

[33] W. Jia, C. Wang, Y. Wang et al., "Qualitative and quantitative analysis of the major constituents in Chinese medical preparation Lianhua-Qingwen capsule by UPLC-DAD-QTOFMS," The Scientific World Journal, vol. 2015, Article ID 731765, 19 pages, 2015.

[34] S. G. Kim, A. Poudel, Y. K. Kim, H. K. Jo, and H. J. Jung, "Development of simultaneous analysis for marker constituents in Hwangryunhaedok-tang and its application in commercial herbal formulas," Journal of Natural Medicines, vol. 67, pp. 390-398, 2013.

[35] D. Tang, D. Yang, A. Tang, and X. Yin, "Simultaneous chemical fingerprint and quantitative analysis of Ginkgo biloba extract by HPLC-DAD," Analytical and Bioanalytical Chemistry, vol. 396, no. 8, pp. 3087-3095, 2010. 

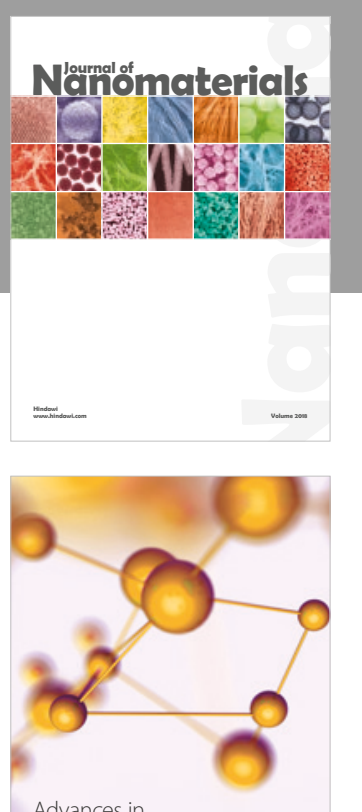

Physical Chemistry
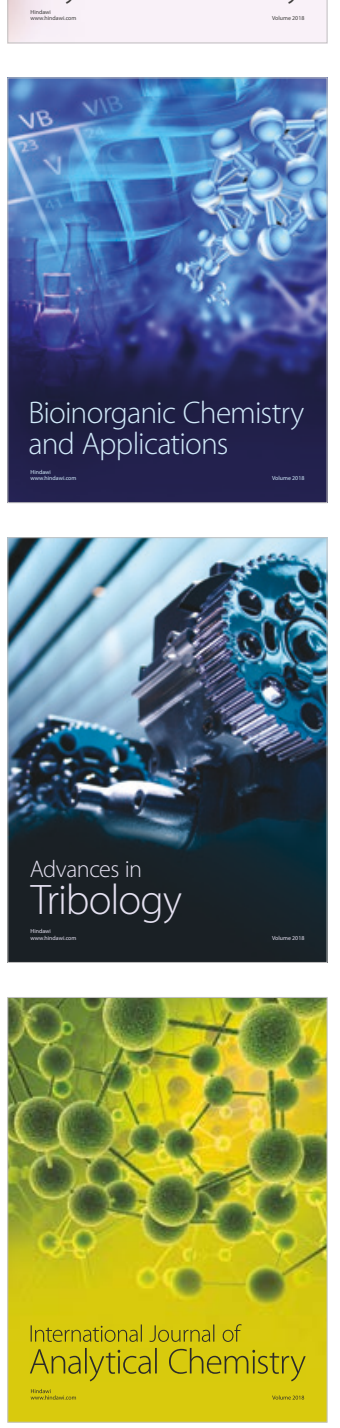

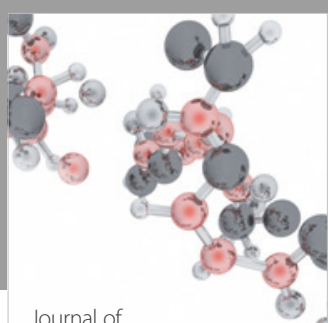

Analytical Methods

in Chemistry

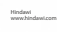

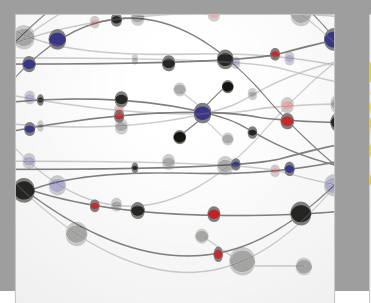

The Scientific World Journal

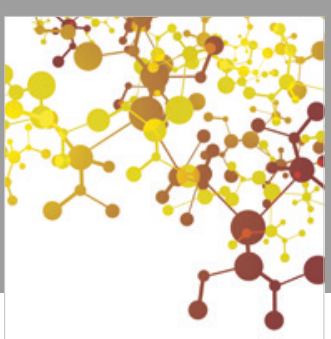

Journal of

Applied Chemistry
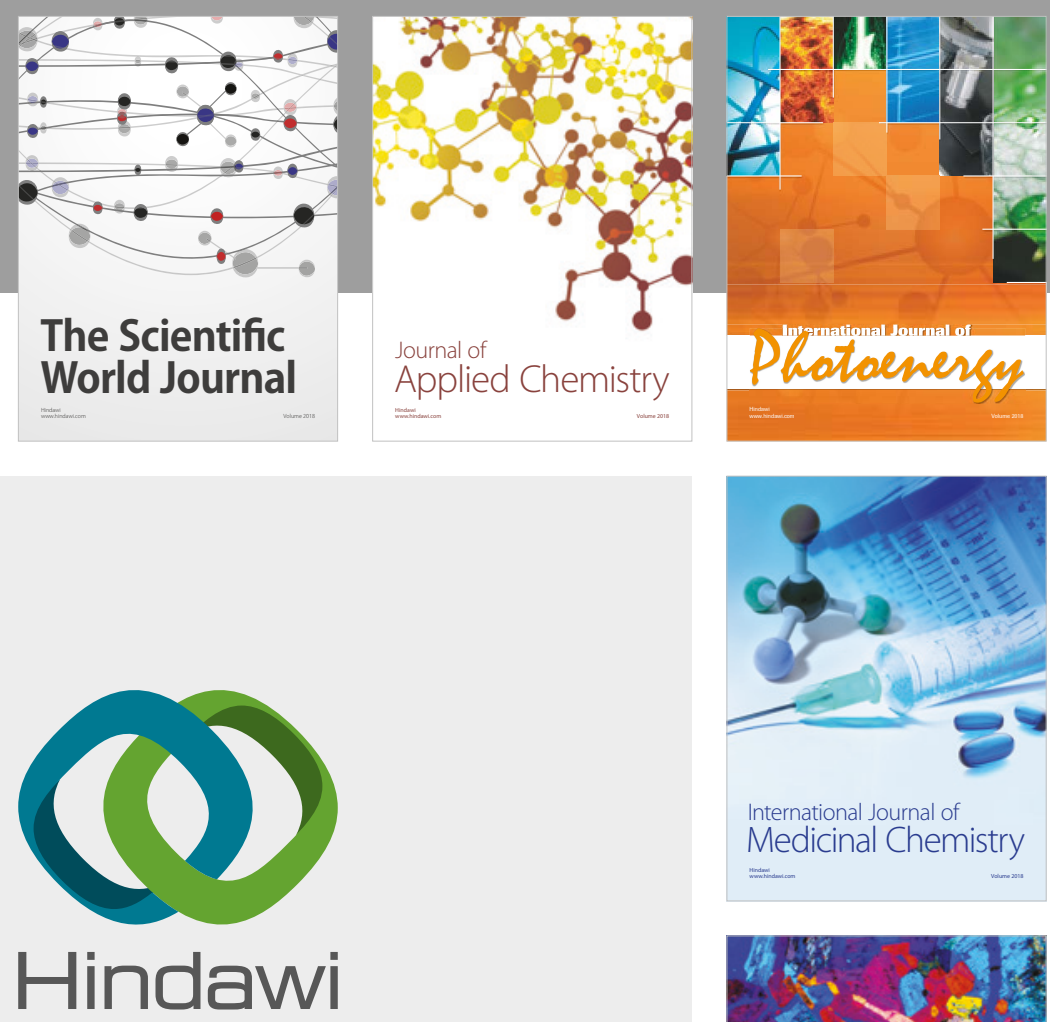

Submit your manuscripts at

www.hindawi.com
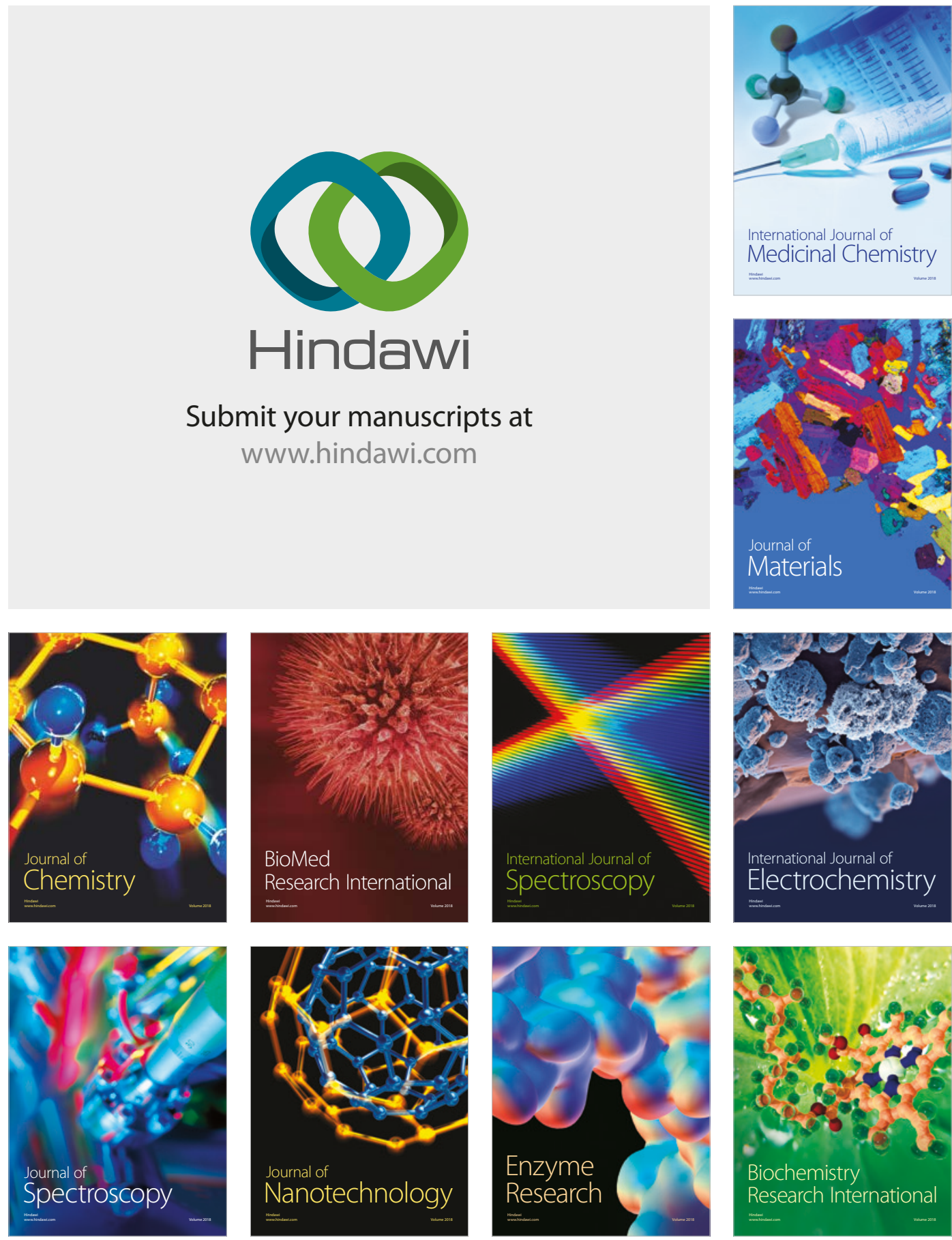
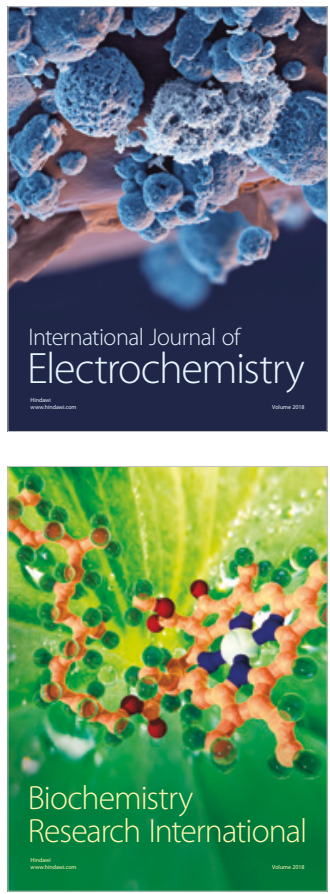